\title{
EML Processing Measurement Techniques
}

\author{
I. Egry', G. Lohöfer' ${ }^{1}$ and D. Matson ${ }^{2}$ \\ ' Institut für Materialphysik im Weltraum, German Aerospace Center, 51170 Cologne, Germany \\ ${ }^{2}$ Department of Mechanical Engineering, Tufts University, Medford, MA 02155, USA
}

\begin{abstract}
Electromagnetic levitation is a useful technique for containerless processing of liquid metals. In combination with non-contact diagnostic tools it can be used for measurements of thermophysical properties of metallic melts. This paper reviews the measurement methods used on ground and in microgravity.
\end{abstract}

Keywords: electromagnetic levitation, thermophysical properties, liquid metals, microgravity, density, surface tension, viscosity, electrical resistivity.

\section{INTRODUCTION}

Thermophysical properties of liquid metals are of central importance for the numerical modelling of industrial solidification, casting and welding processes, and also relevant for wetting phenomena. Most investigated metals and alloys - often technical multicomponent systems with volatile and highly reactive components - have high liquidus temperatures and are prone to evaporation and oxidation problems. These high temperatures and, consequently, high reaction rates, lead to major difficulties in conventional processing of these materials. An elegant way to bypass these problems is the use of containerless methods. Among these, electromagnetic levitation (eml) is the most popular and robust technique for electrically conducting melts. It provides a containerless environment and, at the same time, inductive heating and melting. Containerless processing offers the additional benefit that liquids can be undercooled below their freezing temperatures.
In order to exploit this containerless environment for thermophysical property measurements of liquid metals, eml must be combined with non-contact diagnostic tools. The development and realization of non-contact methods is the major challenge in this field. They are either based on optical or on inductive effects. For example, temperature is measured by pyrometry, using Planck's law of radiation. Density, surface tension and viscosity are measured using high-speed videometry, analyzing the static and dynamic shape of the levitated drop. Electrical resistivity measurements on levitated samples are based on inductive methods.

Although a levitated sample is not in contact with any material container, it is subject to the influence of the levitation forces. Their effect on the measurements is not negligible and must either be taken into account or must be eliminated. In some cases, it is possible to account for the levitation forces quantitatively and, consequently, to correct the measurements, in other cases it is not. Microgravity offers the unique advantage to considerably reduce the strength of the levitation field. In the former case this can be used to verify the mathematical correction formulas by benchmark experiments in space, in the latter case microgravity is an indispensable prerequisite.

\section{ELECTROMAGNETIC LEVITATION}

There exist a number of levitation methods for containerless positioning of samples: açoustic, aerodynamic, electrostatic, and electromagnetic levitation. All of them have certain advantages and drawbacks and, consequently, their specific field of application. For electrically conducting samples, 
electromagnetic levitation is the most widely used technique. It uses an inhomogeneous alternating electromagnetic field to produce a lifting force which compensates the gravitational force. Electromagnetic levitation is intrinsically stable, i.e., the magnetic fields produce a restoring force for deviations of the sample from its equilibrium position. In addition to positioning, the fields also provide inductive heating of the sample.

During the measurements a constant temperature of the molten sample has to be maintained and controlled. This can be achieved partly by adjusting the field strength, but mainly through convective cooling using an inert or slightly reducing gas. Consideration of the oxidation problem is very important, because the formation of oxides on the surface of the liquid sample would lead to erroneous results.

The levitation field $B$ is produced by a levitation coil with a conical or cylindrical shape, connected to an rfgenerator and powered with electrical current of typically $300 \mathrm{kHz}$. This field induces a current in the sample which, in turn, interacts with the field. Levitation is caused by the interaction of the induced current with the levitation field. This can be described as a Lorentz force, $F$. Its magnitude can be expressed, to lowest order in a multipole expansion $/ 1 /$, as:

$$
F=-\frac{\nabla B^{2}}{2 \mu_{0}} \frac{4 \pi}{3} R^{3} Q(q)
$$

where $\mu_{0}$ is the magnetic permeability, $R$ is the radius of the sample, $q=R / \delta$ is a dimensionless quantity and $\delta$ is the skin depth, defined as follows:

$1 / \delta=\sqrt{\frac{\omega \sigma \mu_{0}}{2}}$

Here, $\sigma$ is the electrical conductivity of the sample, and $\omega$ is the frequency of the alternating field. The function $Q(q)$ is given by:

$Q(q)=\frac{2}{4}\left(1-\frac{3}{2 q} \frac{\sinh (2 q)-\sin (2 q)}{\cosh (2 q)-\cos (2 q)}\right)$

Similarly, the power $P$ absorbed by the sample due to ohmic losses of the induced currents is given by $/ 1 /$ :
$P=\frac{B^{2} \omega}{2 \mu_{0}} \frac{4 \pi}{3} R^{3} H(q)$

with $H(q)$ defined as:

$H(q)=\frac{9}{4 q^{2}}\left(a \frac{\sinh (2 q)+\sin (2 q)}{\cosh (2 q)-\cos (2 q)}-1\right)$

The dependence of the functions $Q$ and $H$ on $q$ is shown in Figure 1. Obviously, there is neither levitation nor heating for an insulating sample $(\sigma=0)$; for a perfect conductor $(\sigma=\infty)$, there is levitation, but no heating. The ratio between heating and positioning can be adjusted, within limits, by changing the geometry of the coil (i.e., by changing the field gradient $\nabla B$ ) or by changing the frequency of the power generator (i.e., by changing $q$ ). A complete calculation of the force and heating power exerted on a spherical sample is given in $/ 1-3 /$.

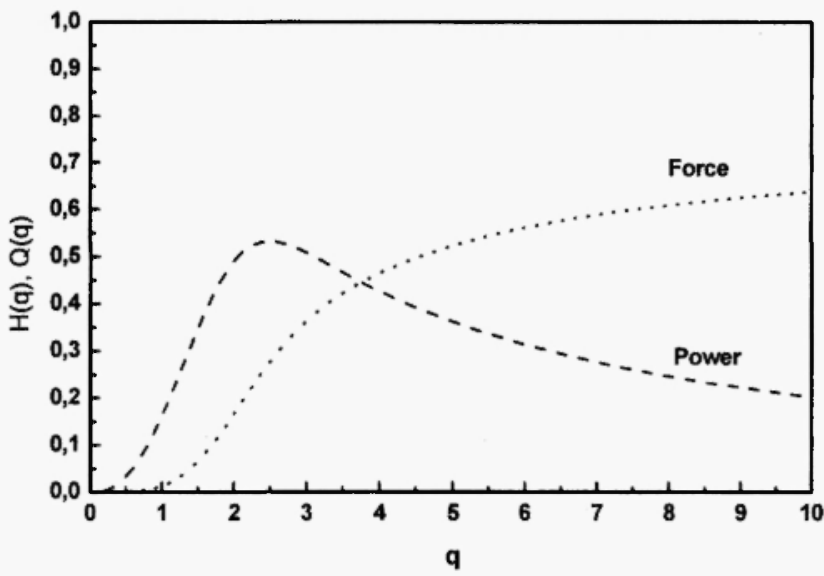

Fig. 1: Positioning and heating efficiency of electromagnetic fields as a function of $\mathrm{q}=\mathrm{R} / \delta$, where $\mathrm{R}$ is the radius and $\delta$ is the skin depth. Dotted line shows $Q(q)$, dashed line $H(q)$.

A levitated sample is positioned in a potential well generated by the electromagnetic fields. Accordingly, a solid sample performs oscillations about its equilibrium position with a frequency which is determined by the "spring constant" of the field, and its mass. If the $\mathrm{z}$ component (parallel to gravity and symmetry axis of the coil) of the electromagnetic field $B_{z}$ varies linearly with $z$, this frequency can be calculated and is given by: /4/ 


$$
\Omega_{\mathrm{II}}^{?}=\frac{3}{2 \rho \mu_{0}}\left(\frac{\partial B_{7}}{\partial z}\right)^{2}, \quad \Omega_{\perp}^{2}=\frac{1}{4} \Omega_{\|}^{2}
$$

where $\Omega_{\|}^{2}$ and $\Omega_{\perp}^{2}$ are the frequencies for oscillations along and perpendicular to the symmetry axis, respectively, and $\rho$ is the sample's density.

Whereas under $\lg$ heating and positioning are provided by the same field and are therefore coupled, they can be decoupled in microgravity. This is due to the fact that only small, omnidirectional positioning forces are required, which are best realised by a quadrupole field. An independent and essentially forcefree heating with high efficiency can be accomplished by a dipole field. Consequently, microgravity eml facilities are equipped with two rf generators, operating independently at different frequencies and providing the required positioning and heating fields.

\section{DENSITY}

The first description of a containerless measurement technique for the density of liquid metals based on electromagnetic levitation is given by Ward $15,6 /$. In order to calculate the density, photographic images of the sample are directly taken from both, the top and the side view position, simultaneously. Only those image pairs are considered for evaluation that exhibit a circular top view profile and a side view profile that is symmetric with respect to the vertical axis. For the successive density calculation, the volume is obtained from the vertical profile using a rotational integral. This method is successfully used by other groups, for instance by Brooks and Quested /7/ who also added a second side-view camera. Densities of electromagnetically levitated metallic samples are also reported by Egry and Gorges /8, 9/ who used a digital CCD camera together with an improved edge detection algorithm.

The above mentioned procedures have the common problem that the sample images are recorded by a camera directly and that, due to the temperature dependent brightness of the sample, the contrast of the images is not constant. This affects the edge detection as shown by Paradis in a systematic investigation $/ 10 /$.
Moreover, if a CCD camera is used, the so-called blooming effect can occur that leads to additional problems with the edge detection $/ 8 /$.

A method that circumvents these kinds of problems is described by Brillo and Egry in $/ 11,12 /$. The optical setup used in their method is schematically shown in Figure 2. The levitated sample is illuminated from the rear by an expanded laser beam. The laser light source (HeNe) is equipped with a beam expander, and a collimator. The collimator is adjusted in such way that the beam is parallel. A lens and a pinhole act together as an optical Fourier filter that removes nonparallel components of the beam which originate at the sample and the windows of the chamber due to scattering and diffraction processes. An interference filter finally removes contributions due to the sample's thermal radiation.

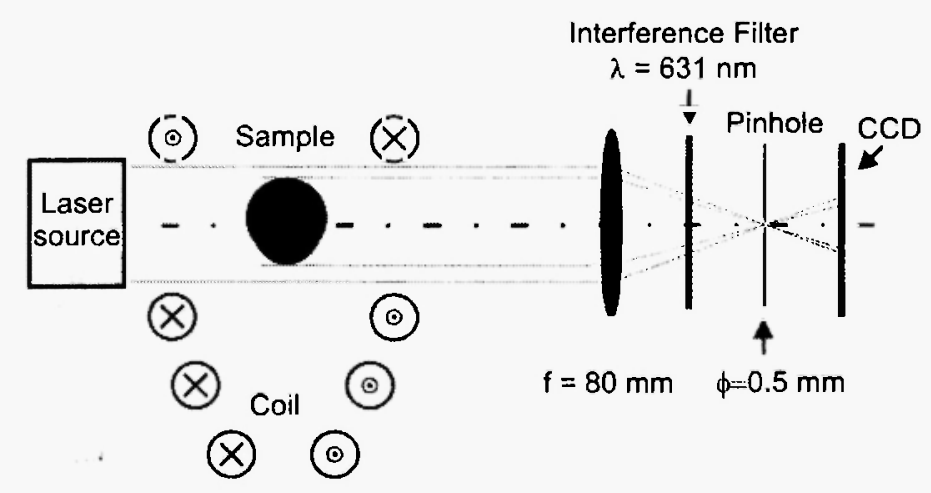

Fig. 2: Schematic diagram of the optical set-up for density measurements.

The shadow image is captured by means of a digital camera, and fed into a computer. It is analyzed by an edge detection abgorithm that locates the edge curve $R(\varphi)$ with $R$ and $\varphi$ being the radius and azimuthal angle with respect to the drop centre. In order to eliminate the influence of oscillations, the edge curve is averaged over typically 1000 frames. It is then fitted by Legendre polynomials of order $\leq 6$ :

$$
\langle R(\varphi)\rangle=\sum_{i=0}^{6} a_{i} P_{i}(\cos (\varphi))
$$

with $P$, being the i-th Legendre polynomial. As shown by a thorough analysis of top view images in $/ 11 /$, the 
equilibrium shape of the sample can be assumed to be symmetrical with respect to the vertical axis. Hence, the volume is calculated using the following integral:

$$
V_{P}=\frac{2}{3} \pi \int_{0}^{\pi}<R(\varphi)>^{3} \sin (\varphi) d \varphi
$$

$V_{\mathrm{p}}$ is the volume in pixel units. It is related to the real volume $V$ according to $V=q V_{p}$, with $q$ being the scaling factor. The scaling factor $q$ of the system is determined by a calibration procedure. With $M$ being the mass of the sample, the density, $\rho$, is calculated from $\rho=M / V$.

\section{SURFACE TENSION}

The surface tension of levitated liquid drops can be measured using the oscillating drop technique. This technique is based on the fact that surface tension acts as a restoring force for surface oscillations (capillary waves) of liquid drops. The deformation of the sample's shape due to the surface oscillations can be described by spherical harmonics $Y_{n}{ }^{m}$ :

$$
R(\vartheta, \varphi, t)=\sum_{n} \sum_{m=-n}^{m=+n} a_{n \cdot m}(t) \cdot Y_{n}^{m}(\vartheta, \varphi)
$$

Here, $R$ is the time- and angle-dependent radius of the sphere, $n>0$ and $|m|<n$ are the indices of the spherical harmonics. By digital image processing and Fast Fourier Transformation (FFT) of the oscillations of the sample around the equilibrium shape and equilibrium position one obtains the characteristic spectra and, consequently, the frequencies of both, the surface oscillations and the sample translations.

According to Rayleigh /13/ the relation between the surface tension $\gamma$ of a non-rotating spherical sample and the characteristic surface oscillation frequencies $\omega_{n m}$ is given by the Rayleigh law:

$\omega_{\mathrm{n}, \mathrm{m}}^{2}=\mathrm{n}(\mathrm{n}+2)(\mathrm{n}-1) \cdot \frac{4 \pi}{3} \frac{\gamma}{M}$

where $M$ is the mass of the sample. It is the only additional parameter necessary to determine the surface tension $\gamma$.
In the case of spherical symmetry, the frequencies do not depend on the index $m$. Due to the incompressibility of the liquid, the mode $n=0$ does not appear, and the mode $n=I$ corresponds to a translational oscillation of the entire sphere, without distorting its surface. Consequently, the fundamental mode is $n=2$. This is the lowest surface oscillation mode, and its frequency is called Rayleigh-frequency $\omega_{R}:$

$\omega_{\mathrm{R}}{ }^{?}=\frac{32 \pi}{3} \cdot \frac{\gamma}{M}$

For sample masses around $\lg , v_{R}=\omega_{R} / 2 \pi$ is typically of the order of $40 \mathrm{~Hz}$.

For a non-rotating spherical droplet this mode is 5fold $(2 n+1-$ fold $)$ degenerate:

$\omega_{2, m}=\omega_{2}=\omega_{R}$

Oscillation modes with $n>2$ are well separated due to their distinctly higher frequencies and are often not visible, because of their strong damping.

Under experimental conditions on earth, different forces act on the liquid sample, causing an aspherical equilibrium shape. Consequently, the Rayleigh frequency $\omega_{R}$ for the different surface oscillation modes of the liquid sample splits up into three unequally spaced peaks.

Cummings and Blackburn $/ 4 /$ investigated this problem theoretically and derived formulae for calculating the Rayleigh-frequency $\omega_{k}$ for $n=2$ from the shifted oscillation frequencies $\omega_{2 m}$ :

$$
\begin{aligned}
& \omega_{20}^{2}=\omega_{R}^{2}+\overline{\Omega_{t r}^{2}} \cdot\left(3.832-0.1714 \cdot\left(\frac{z_{0}}{R}\right)^{2}\right) \\
& \omega_{2.1}^{2}=\omega_{R}^{2}+\overline{\Omega_{t r}^{2}} \cdot\left(3.775+0.5143 \cdot\left(\frac{z_{0}}{R}\right)^{2}\right) \\
& \omega_{2,2}^{2}=\omega_{R}^{2}+\overline{\Omega_{t r}^{2}} \cdot\left(-0.9297+2.571 \cdot\left(\frac{z_{0}}{R}\right)^{2}\right)
\end{aligned}
$$

Here $\omega_{2, m}$ are the shifted surface oscillation frequencies for $n=2 ; \overline{\Omega_{t r}^{\hat{\imath}}}$ denotes the mean quadratic translation 
frequency (of the 3-dimensional sample translations), $m$ is the index of the surface oscillation-modes, and $R$ is the radius of the sample. Finally,

$$
z_{0}=\frac{g}{2 \cdot \overline{\Omega_{t r}^{2}}}
$$

where $g$ is the gravitational acceleration.

In addition, sample rotation along the symmetry axis lifts the degeneracy of $+m$ and $-m$ modes $/ 14 /$, according to the relation:

$\omega_{2, m}^{r o t}=\omega_{2, m}^{0}+\frac{m}{2} \cdot \Omega_{r o t}$

with $\Omega_{r o t}$ being the rotational frequency.

Consequently, the degeneracy of the oscillation mode $n=2$ is completely removed and five oscillation frequencies for $m=-2,-1,0,+1,+2$ are distinguishable.

In case that all oscillation frequencies are visible in the characteristic spectra, a sum-formula derived by Cummings and Blackburn $/ 4 /$ can be used:

$$
\begin{aligned}
\omega_{\mathrm{R}}^{2}= & \frac{1}{s} \sum_{m} \omega_{2, \mathrm{~m}}^{2}-1.9 \cdot \overline{\Omega_{t r}^{2}}- \\
& \left.0.3 \cdot \overline{\left(\Omega_{t r}^{2}\right.}\right)^{-1} \cdot\left(\frac{g}{R}\right)^{2}
\end{aligned}
$$

The last two terms, containing $\Omega_{t r}$, are a correction of the order $5-10 \%$ to the original Rayleigh formula.

The surface oscillations of the liquid droplet are recorded from the top-position - viewing along the symmetry axis - by a digital high-speed-camera and stored by a high-speed-PC. Sampling rate and exposure time are governed by the uncertainty relation: $T \Delta v=1$, where $T$ is the measuring time and $\Delta v$ the error in frequency determination, and by the Nyquist theorem 115/: $v_{o b s}>2 v_{\max }$, which ensures that aliasing effects are avoided when the sampling rate $v_{o b s}$ is at least twice the maximum frequency $v_{\max }$ appearing in the measured spectrum.

Using a digital image processing-system, first the area of the sample's image and the position of its centre of mass are evaluated to determine the three sample translation frequencies $\Omega_{r r}$. Subsequently, two perpendicular radii of the sample's image are determined for each frame and their sum as well as their difference is calculated. With the help of selection rules $/ 16,17 /$ it is then possible to associate the characteristic frequencies $\omega_{2, m}$ in the spectra with the individual oscillation modes. The oscillation modes with $n=2$ and $m- \pm 2$ are suppressed in the FFT of the sum-signal, while in the FFT of the difference signal the oscillation mode with $n=2$ and $m=0$ is suppressed. This is schematically shown in Figure 3, where different sample oscillations are shown as viewed along the symmetry axis. For the projection of a symmetric oscillation-mode, like: $n=2 / m=0$, the radius does not depend on $\varphi$, and so the difference of perpendicular radii vanishes. Consequently, the $n=2 / m=0$ oscillation mode does not contribute to the FFT spectrum of the difference signal. In the projection of the $n=2 / m=2$ mode the deviations of perpendicular radii from a circle cancel each other exactly. So, for the $n=2 / m=2$ oscillation mode the sum of perpendicular radii is a constant. Because constant signals do not possess Fourier components with $\omega \neq 0$, the $n=2 / m=2$ oscillation mode is suppressed in the FFT spectrum of the sum-signal.

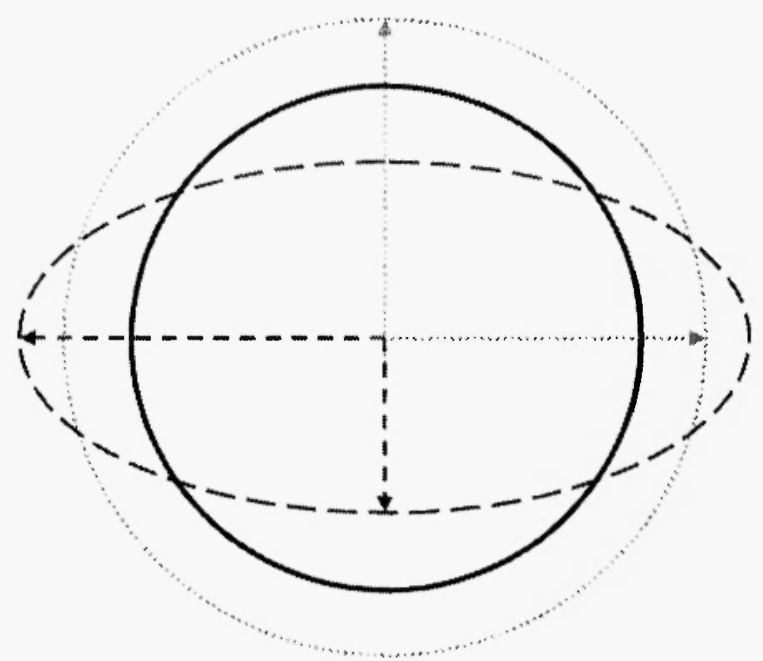

Fig. 3: Schematic drawing of different sample deformations. The equilibrium shape is shown by the full line. The dotted line shows a symmetric deformation, the dashed line shows an elliptical deformation. The arrows indicate pairs of perpendicular radii. 
From the above discussion, the advantage of performing the measurement under $\underline{\mu} \underline{\underline{g}}$ is obvious: no Cummings correction is necessary; consequently, no mode analysis needs to be performed. Instead, Eq. 11 can be applied directly. Different oscillation spectra, taken under $1 \mathrm{~g}$ and $\mu \mathrm{g}$ conditions on the same sample, are shown in Figure 4.

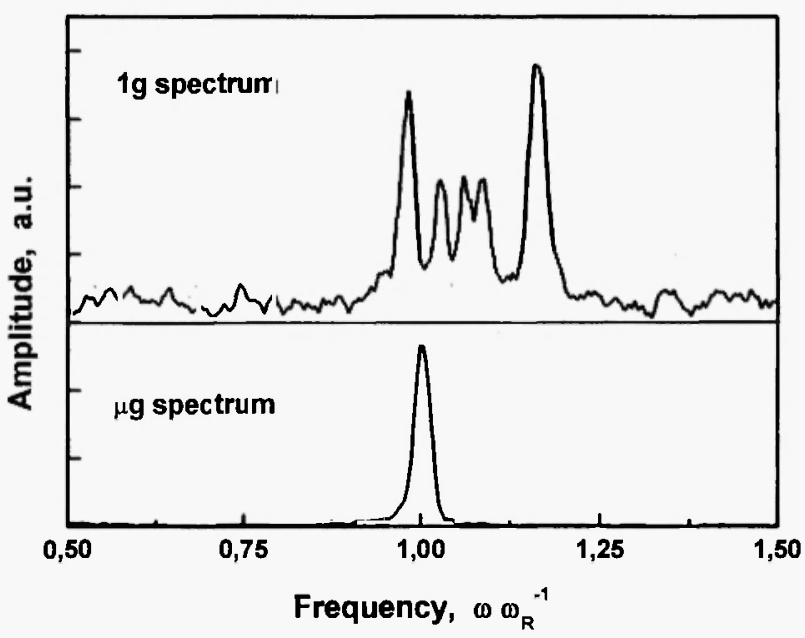

Fig. 4: Oscillation spectrum of a liquid $\mathrm{Au}-\mathrm{Cu}$ drop recorded under terrestrial (top) and microgravity (bottom) conditions.

\section{ELECTRICAL RESISTIVITY}

The presence of high frequency magnetic fields in the eml suggests the use of these fields simultaneously also for a contactless inductive determination of the electrical resistivity of the levitated metal droplet.

The alternating magnetic levitation field $B_{\text {coil }} \propto I_{\text {coil }}$ generated by the current $I_{\text {coil }}$ in the levitation coil induces a voltage in the levitated sample. This voltage drives eddy currents $I_{s}\left(\rho_{s}, r_{s}\right)$ in the sample, the strength of which depends on the resistivity $\rho_{s}$ and the radius $r_{s}$ of the liquid material. The eddy currents again generate an additional alternating magnetic field $B_{s}\left(\rho_{s}, r_{s}\right) \propto I_{s}\left(\rho_{s}, r_{s}\right)$. Both magnetic fields finally induce a voltage $U_{\text {coil }}$ in the secondary measurement coil described by the complex equation

$\left|U_{\text {coil }}\right| e^{i \varphi}=\left(Z_{\text {coil }}+Z_{s}\left(\rho_{s}, r_{s}\right) \cdot\left|I_{\text {coil }}\right|\right.$
The equivalent electrical circuit is shown in Figure 5. By a measurement of the absolute values of $U_{\text {coll }}, I_{\text {coil }}$ and the phase difference $\varphi$ between current and voltage, the (complex) impedance $Z_{\text {coil }}+Z_{s}\left(\rho_{s}, r_{s}\right)$ can be determined. After a calibration measurement without sample, which yields the offset $Z_{\text {coil }}$, this yields the real and imaginary value of the sample impedance $Z_{s}\left(\rho_{s}, r_{s}\right)$, from which the two unknown sample properties $\rho_{s}$ and $r_{s}$ can be determined, provided that the physical model connecting the measured sample impedance $Z_{s}$ with the thermophysical properties $\rho_{s}$ and $r_{s}$ is well known $118 \%$.

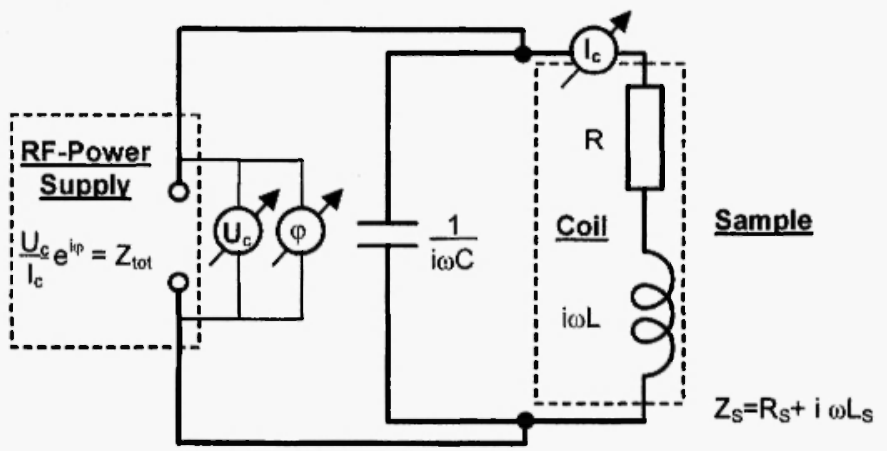

Fig. 5: Block diagram of a levitation facility together with the inductively coupled sample and the connected measurement equipment.

Besides $\rho_{s}$ and $r_{s}$, the sample impedance $Z_{s}$ is also influenced by the shape of the liquid levitated sample and the shape of the surrounding magnetic levitation field, which are both generally not very well known. This is different, however, if samples are levitated in the forceless microgravity environment. Due to the very low magnetic positioning field, necessary to keep the sample inside the experimental area of the levitation facility, the liquid sample assumes an almost spherical shape. Furthermore, the heating field, which can simultaneously be used as measurement field, is almost homogeneous near the sample. Both advantages, due to the microgravity environment, allow a very precise determination of the electrical resistivity $\rho_{s}$ of liquid metals.

Measurement of the electrical resistivity of $\mathrm{CO}_{80} \mathrm{Pd}_{20}$ have been performed under microgravity in the 
electromagnetic levitation facility TEMPUS. The results are shown in Figure 6. Above ca. $1350 \mathrm{~K}$ in the solid and ca. $1370 \mathrm{~K}$ in the liquid state, the mean values of the resistivity data of $\mathrm{Co}_{80} \mathrm{Pd}_{20}$ exhibit perfect linear temperature dependence. Below these temperatures the data points deviate at first weakly and then strongly from this behaviour, indicating the onset of a magnetic ordering. In this temperature range the data points may no longer be interpreted as resistivities, because the applied inductive measurement technique reacts also very sensitively to ferromagnetic sample properties.

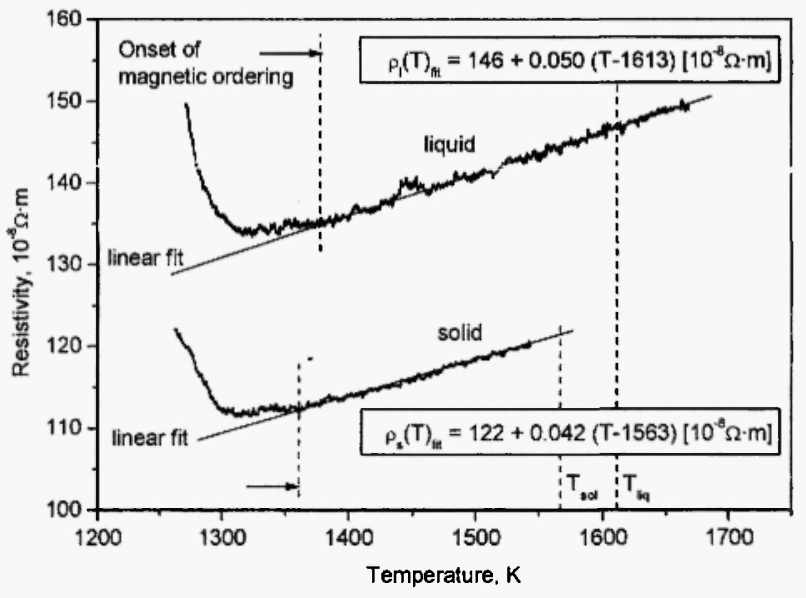

Fig. 6: Mean values of the electrical resistivity data for $\mathrm{Co}_{80} \mathrm{Pd}_{20}$ in the solid and liquid state, together with the corresponding linear fits as function of the temperature. $T_{\mathrm{v} o l}$ and $T_{l i q}$ indicate the solidus and liquidus temperatures, respectively. Due to the onset of magnetic ordering in this alloy, the data points represent the electrical resistivity only above ca. $1370 \mathrm{~K}$.

\section{VISCOSITY}

For viscosity measurements, using electromagnetic levitation, the oscillating drop technique can also be applied. In this case however, the damping of the oscillations needs to be determined. This damping $\Gamma$ can be related to the sample's viscosity $\eta$ according to

$\Gamma=\eta \frac{20 \pi}{3} \frac{R_{0}}{M}$

where $M$ is the mass and $R_{0}$ the radius of the drop. This formula was derived by Chandrasekhar/19/ and is valid for spherical samples in the absence of external forces. In terrestrial electromagnetic levitation a strong inhomogeneous field is necessary to compensate gravity. This strong field not only introduces a magnetic pressure and, consequently, a deformation of the liquid drop (which leads to a modified frequency spectrum, i.e., an effective surface tension, as discussed in section 4), but it also affects the fluid flow inside the sample. In essence, the magnetic field $B$ tends to damp any fluid flow perpendicular to it. This influence can be crudely described by an additional magnetic viscosity $\eta_{\text {mag }} / 20 /$

$\eta_{\text {mag }}=\sigma B^{2} R^{2}$

where $\sigma$ denotes the electrical conductivity, $B$ the magnetic field, and $R$ the radius of the sample. For high Reynolds numbers $R e$

$\operatorname{Re}=\frac{R^{2} \rho}{\eta} \sqrt{\frac{8 \gamma}{\rho R^{3}}}=5 \frac{\omega_{R}}{\Gamma_{C}}$

Bratz and Egry /21/ derived correction formulas to Chandrasekhar's result, Eq. 20, similar to the Cummings correction (Eq. 13-15). They are valid for laminar flow only, and do not describe the experimental situation correctly. Consequently, there exist no analytical expressions for comparison with experimental data. However, the problem has been approached numerically by Bardet $122 \%$. He finds that turbulent damping dominates for Reynolds numbers $R e>30$, rendering the measurement of viscosity impossible.

The ratio between the sample viscosity, $\eta$, and the magnetic viscosity $\eta_{\text {mag }}$, is called Hartmann number /20/:

$H_{a}^{2}=\frac{\eta_{\text {mag }}}{\eta}$ with $H_{a}=\sqrt{\frac{\sigma}{\eta}} B R$

For viscosity measurements, one requires $H_{a} \ll 1$. This condition is violated in terrestrial levitation. For example, $H_{a} \approx 4$ for nickel $/ 22 /$

In order to measure the damping, one can either detect the free decay of an oscillation, excited by a single pulse, or one may induce forced oscillations with variable frequency and determine the resonant response 
of the system. In a noisy environment, like in terrestrial eml, surface oscillations are stochastically self-excited, and the free decay cannot be observed. Therefore, forced oscillations of the sample's surface are induced by modulating the amplitude of the levitation field $\left(\omega_{H}\right)$ via a frequency generator with a low frequency $\omega_{m}$ :

$B=B_{0} \sin \left(\omega_{H} t\right)\left(1+\varepsilon \sin \left(\omega_{m} t\right)\right)$

The frequency of the $\mathrm{rf}$-field is in the range of 300 $\mathrm{kHz}$, while the resonance frequencies of the used samples were about $35-50 \mathrm{~Hz}$.

By modulating the external field the radius of the sample is forced to perform oscillations with a frequency $\omega_{m}$, and the motion of the radius can be described by the differential equation:

$\ddot{x}+2 D \omega_{0} \dot{x}+\omega_{0}^{2} x=F_{0} \cos (\chi \tau)$ with $\chi=\frac{\omega_{n}}{\omega_{0}}$

and $\tau=\omega_{0} t$

where $\omega_{0}$ is the resonance frequency of the drop oscillations $\left(\omega_{0} \approx \omega_{R}\right)$. The amplitude of the forced oscillation is given by the equation:

$$
A=A_{0} \frac{1}{\sqrt{\left(1-\chi^{2}\right)^{2}+4 D^{2} \chi^{2}}}, \quad D=\frac{\Gamma}{\omega_{0}}
$$

where $D$ is the parameter which describes the damping. A fit of the amplitude of the sample oscillations in dependence of the modulation frequency yields the resonance function, Eq. 26, from which the damping constant can be derived.

Due to the geometry of the coil mainly the $m=0$ oscillation mode (see section 4 of surface oscillations) is excited and due to the rotation of the sample the resonance frequencies of the different oscillation modes are shifted away from the $m=0$ mode so that they do not affect the measurement at the resonance frequency of $m=0$. An example of the oscillation amplitudes as a function of the excitation frequency is shown in Figure 7, taken from $/ 23 /$.

Consequently, the oscillating drop technique cannot be used for viscosity measurements in eml on ground. The situation might be different in electrostatic levitation (esl), where there is less turbulent flow in the sample.

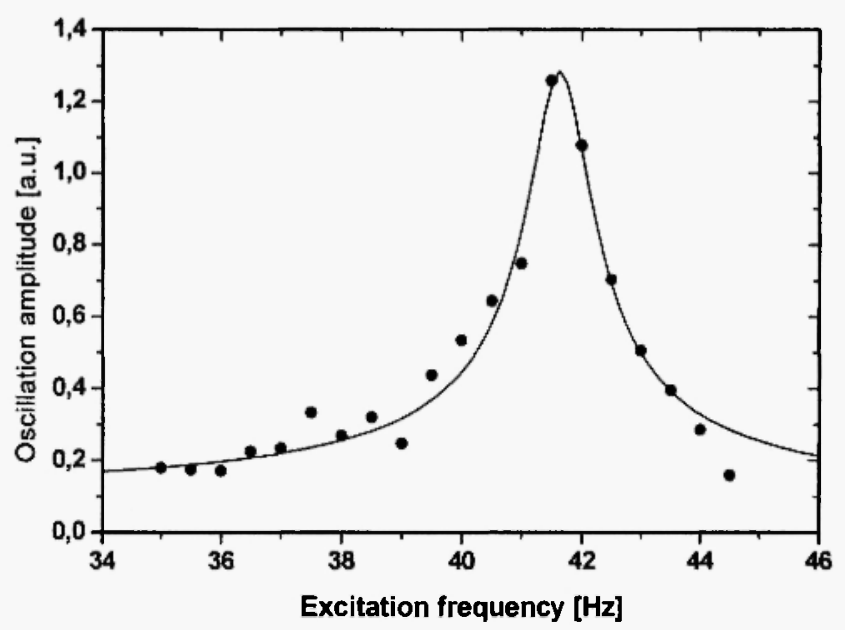

Fig. 7: Measured oscillation amplitudes as a function of excitation frequency and fitted resonance function for a Nickel sample at $1620 \mathrm{~K}$.

Under $\mu \mathrm{g}$ conditions this method can be applied, and has been successfully used in Spacelab and parabolic flight experiments. It must be noted, however, that in such experiments the heating field must be switched off to take full advantage of the small positioning field. In other words, the experiments have to be carried out in a transient, rather than an isothermal mode.

\section{SPECIFIC HEAT}

A non-contact method developed by Fecht and Johnson $/ 24$ / can be used to determine the specific heat in levitation experiments. It is a variant of non-contact modulation calorimetry, normally used in low temperature physics. The heater power is modulated according to $P_{\omega}(t)=\Delta P_{\omega} \cos (\omega t)$ resulting in a modulated temperature response $\Delta T_{\omega}$ of the sample. A thermal model considering heat loss to the environment and heat conduction within the sample has been developed by Wunderlich $25 \%$. It considers heat loss to the exterior (typically by radiation only if the experiment is carried out under vacuum), spatially inhomogeneous heating of the sample, and heat conduction within the sample. For small Biot numbers, $B i=k_{r} k_{c}<<1$, where $k_{r}$ is the heat loss due to radiation and $k_{c}$ the heat loss due to heat conduction, adiabatic conditions are realized and quantitative modulation calorimetry is possible. Under such conditions, the following relation for $\Delta T_{\omega}$ is obtained: 
$\Delta T_{\omega}=\frac{\Delta P_{\omega}}{\omega c_{p}}\left\{\left(1+\left(\frac{\omega}{\dot{\lambda}_{1}}\right)^{2}\right)\left(1+\left(\frac{\lambda_{2}}{\omega}\right)^{2}\right)\right\}^{-1 / 2}$

where $\lambda_{1}$ and $\lambda_{2}$ are functions of $k_{r}$ and $k_{c}$ such that for $B i \ll 1, \lambda_{2} \ll \lambda_{1}$. This allows to choose the modulation frequency between $\lambda_{2}$ and $\lambda_{1}$ such that $\lambda_{2} \ll \omega<\lambda_{1}$. Under these circumstances, a simple relation for the the specific heat, $c_{p}$, can be derived:

$c_{p}=\frac{1}{\omega} \frac{\Delta P_{\omega}}{\Delta T_{r,}}$

The power input into the sample, $\Delta P_{\omega}$, cannot be measured directly. It is related to the current $I_{\omega}$ flowing through the heating coil by a coupling coefficient $G_{H}$ which has to be determined separately:

$\Delta P_{\omega}=G_{H} I_{\omega}^{2}$

It should be noted that a harmonic modulation of the current with $\omega^{\prime}$ leads to components in the power $P$ with $\omega=0, \omega=\omega^{\prime}$ and $\omega=2 \omega^{\prime}$. The static and frequencydoubled components can also be analyzed along the lines indicated above.

Modulation calorimetry needs to be carried out under isothermal conditions, i.e., the sample must be allowed to thermalize at a predefined temperature before the modulation signal can be applied. Therefore, such experiments cannot be performed during parabolic flights. Fecht and co-workers $/ 26 /$ applied this method during the MSL-1 Spacelab mission. More recently, Wunderlich et al., performed modulation calorimetry during a sounding rocket experiment on a Ti46Al8 Nb alloy in the framework of the IMPRESS project $/ 27 /$. It was possible to determine both, the specific heat, and the total hemispherical emissivity in the liquid phase $128 \%$.

\section{SOLIDIFICATION VELOCITY}

When a liquid solidifies, it releases the heat of fusion. If solidification occurs from an undercooled melt, it causes a localized rapid temperature rise at the solid-liquid interface, called recalescence. The interface region contains the growing dendrites and retained interdendritic liquid at the solidus temperature, with the solid-liquid ratio defined by the relative undercooling. Dendrite growth velocity measurement is accomplished by tracking the motion of this thermal front since the position of the front is readily seen as a sharply defined change in radiance $/ 29$ / moving rapidly across the exterior surface of the sample. The front appears macroscopic in scale and is composed of an array of growing microscopic dendrite tips. Dendrite growth morphology ranges from an angular frontal growth pattern arising from an array of dendrites sharing a common nucleation point $/ 30 /$ to a spherical seaweed growth pattern $/ 31 /$ to faceted growth along preferred orientations 132 . The selection of the morphology depends not only on sample composition but also on undercooling. The transition from oriented growth to spherical growth occurs near the critical undercooling where dendrite growth theory diverges from experimental observations /33/ and remains an area of active research investigation.

Walker $/ 34 /$ was the first to measure the growth velocity of nickel as a function of undercooling using a vitreous silica boat and two photodiodes located a set distance apart. When the front passed by each photodiode, the signal was observed to rise significantly due to the temperature increase and the velocity could then be determined from knowledge of the travel distance and rise time difference. Cinematography was later used $/ 35 /$ to track the motion of the thermal front with contrast developed by changes in thermal radiance and changes in emissivity with phase; in each image, cool regions appear dark and hot regions appear light.

Taking advantage of levitation processing techniques to avoid influencing dendrite motion by crucible interaction, a capacitance trigger was used to stimulate nucleation at a known location and observe when the front passes by a pair of wide-angle photodiodes $/ 36 /$. With a large region under observation, not only the time lag between photodiodes but also the rise time over the known viewing angle could be used to verify the accuracy of the growth measurement. This technique solved the problem of defining when and where nucleation occurred but still did not address whether the front motion and dendrite tip velocity were one and the same.

Since these techniques relied on observations of the dendrite growth along the surface of the sample and 
since the orientation of the dendrite growth direction may not be parallel to the surface, new techniques were developed to track motion through the center of the sample. By combining triggering and digital cinematography on levitated samples $137,38 /$ researchers were able to define when and where both nucleation and reappearance of the front when it intersects the opposing surface in order to define the absolute path length and timing of growth through the sample interior $/ 39 /$.

For a typical electromagnetically levitated sample the diameter is on the order of $8 \mathrm{~mm}$. With solidification velocities on the order of $1-70 \mathrm{~m} / \mathrm{s} / 33 /$ this corresponds to a solidification time of 8.0-0.1 milliseconds requiring sampling rates of at least $250-17500 \mathrm{~Hz}$. By combining cinematography, nucleation triggering and high speed photodiode sensing, researchers can correct for differences in path length due to the significant gravityinduced surface deformation in ground-based investigations. It is, however, not possible to reduce the convection induced by the levitation field which results in internal recirculation loops with a characteristic maximum velocity on the order of $0.32 \mathrm{~m} / \mathrm{s} / 40 /$ with a loop circulation rate of around $20 \mathrm{~Hz}$ and a Reynolds number around 2800 .

In space, the spherical nature of the sample eliminates the need for these corrections and allows the researcher to take advantage of the associated reduction in induced sample convection. A wide range of flow conditions may now be accessed by careful selection of the levitation positioning and heating field configuration. Maximum recirculation velocities may be selected from within the range $0.03-0.16 \mathrm{~m} / \mathrm{s}$ corresponding to Reynolds numbers of 100-1700/40/. With this wide range in flow conditions available, research on the influence of convection on transformation kinetics and phase selection is now possible including both eutectic $/ 41 /$ and peritectic $/ 42$, 43 / systems of commercial importance.

\section{SUMMARY}

Electromagnetic levitation is a useful tool for measuring thermophysical properties of liquid metals, especially at high temperatures. If the effect of the levitation field is included in the working equations, the results are precise and reliable. This has been proven by benchmark experiments in microgravity. This technique is complementary to aerodynamic and electrostatic levitation in terms of temperature range, processing environment and materials which can be processed. In fact, this suite of levitation techniques allows studying virtually any (inorganic) material, oxide, insulator, metal, from room temperature up to $3000 \mathrm{~K}$.

Microgravity offers additional advantages as demonstrated during two Spacelab missions, several parabolic flight campaigns, and two recent sounding rocket flights. The full strength of this environment can only be exploited with sufficient experiment time, i.e., on board the International Space Station ISS. The MSLEML (Materials Science Lab - Electro Magnetic Levitator) facility is presently under construction and will become operational in 2011. It may then become feasible to establish a Global Institute of Standards for Thermophysical Properties on board the ISS.

\section{ACKNOWLEDGEMENTS}

I.E and G.L. gratefully acknowledge financial support by ESA through the ThermoLab project (ESA MAP AO-99-022 contract 14306/01/NL/SH). D.M. gratefully acknowledges financial support by NASA through NASA Grant NNX08AL21G.

\section{REFERENCES}

1. G. Lohöfer, SIAM J. Appl. Math., 49, 567-581 (1989).

2. G. Lohöfer, Quarterly Appl. Math., 11, 495-518 (1993).

3. G. Lohöfer, IMA J. Appl. Math., 68, 1-21 (2003).

4. D. Cummings and D. Blackburn, J. Fluid Mech., 224, 395-416 (1991).

5. A.E. El-Mehairy and R. G. Ward, Trans. Met. Soc. AIME., 227, 1226-1228 (1963).

6. S.Y. Shiriashi and R.G. Ward, Can. Met. Quat., 3, 117-122 (1964).

7. R.F. Brooks, B. Monaghan, A.J. Barnicoat, A. McCabe, K.C. Mills and P.N. Quested, Int. J. Thermophys. 17, 1151-1161 (1996).

8. E. Gorges, L. Racz, A. Schillings and I. Egry, Int. J. Thermophys., 17, 1163-1172 (1996). 
9. G. Jacobs, I. Egry, E. Gorges and M. Langen, Int. J. Thermophys. 19, 895-903 (1998).

10. P.F. Paradis, J. Yu, T. Ishikawa, T. Aoyama, S. Yoda and J.K.R. Weber, J. Cryst. Growth, 249, 523-530 (2003).

11. J. Brillo and I. Egry, Int. J. Thermophys., 24, 11551170 (2003).

12. J. Brillo and I. Egry, Z. Metallkd., 95, 691-697 (2004).

13. Lord J.S.W. Rayleigh, On the capillary phenomena of jets, Proc. Roy. Soc., 29, 71-97 (1879).

14. F.H. Busse, J. Fluid Mech., 142, 1-8 (1984).

15. A.V. Oppenheim and R.W. Schafer, Digital Signal Processing, Prentice-Hall, Inc., Englewood Cliffs, New Jersey (1975).

16. S. Sauerland, K. Eckler and I. Egry, J. Mat. Sci. Letters, 11, 330-333 (1992).

17. I. Egry and S. Sauerland, Mat. Sci. and Eng., A178, 73-76 (1994).

18. G. Lohöfer, Meas. Sci. Technol., 16, 417-425 (2005).

19. S. Chandrasekhar, Hydrodynamic and Hydromagnetic Stability, Dover, New York, (1981).

20. J. Jackson, Classical Electrodynamics, Wiley, New York (1967).

21. A. Bratz and I. Egry, J. Fluid Mech., 298, 341-359, (1995).

22. B. Bardet, Levitation Electromagnetique Experiences terrestres et simulations numeriques, $\mathrm{PhD}$ thesis, Institut National Polytechnique de Grenoble, France (2006) - in French.

23. S. Schneider, Viskosität unterkühlter Metallschmelzen, PhD thesis, RWTH Aachen, Germany (2002) - in German.

24. H.J. Fecht and W. Johnson, Rev. Sci. Instr., 62, 1299-1303 (1991)

25. R. Wunderlich and H.J. Fecht, Meas. Sci. Tech., 16, 402-416 (2005).

26. R. Wunderlich, R. Sagel, Ch. Ettel, H.J. Fecht, D. Lee, S. Glade and W. Johnson, Measurement o thermophysical properties of bulk metallic glass forming liquid alloys under reduced gravity conditions, in: Solidification 1999, W. Hofmeister, J. Rogers, N. Singh, S. Marsh, P. Vorhees, eds., TMS, Warrendale PA, 53-64 (1999).
27. D. Jarvis and D. Voss, Mat.Sci. Eng., A413-414, 583-591 (2005).

28. R. Wunderlich, private communication (2007).

29. B. Bassler, W. Hofmeister, G. Carro and R. Bayuzick, Met. Trans. A, 25A, 1301-1307 (1994).

30. Y. Wu, T. Piccone, Y. Shiohara and M. Flemings, Met. Trans. A, 18, 915-924 (1987).

31. A. Mullis, K. Dragnevski and R. Cochrane, Mat. Sci. Eng., A375, 157-162 (2004).

32. T. Aoyama and K. Kuribayashi, Acta Mater., 48, 3739-3744 (2000).

33. D. Herlach, R. Cochrane, I. Egry, H.J. Fecht and A. Greer, Int. Mat. Rev., 38, 273-347 (1993).

34. J. Walker, in: Principles of Solidification, B. Chalmers, ed., John Wiley \& Sons, New York, NY, 112 (1964)

35. A. Colligan and B. Bayles, Acta Metall., 10, 895897 (1962).

36. K. Eckler, M. Kratz and I. Egry, Rev. Sci. Instrum., 64, 2639-2642 (1993)

37. J. Lum, D. Matson and M. Flemings, Met. Trans. $B, 27 B, 865-870$ (1996).

38. B. Bassler, R. Brunner, W. Hofmeister and R. Bayuzick, Rev. Sci. Instrum., 68, 1846-1852 (1997).

39. D. Matson, The measurement of dentrite tip propagation velocity during growth in undercooled metallic melts, in: Solidification 1998, S. Marsh, et al., eds., TMS, Warrendale PA, 233-242 (1999).

40. R. Hyers, D.M. Matson, K. Kelton and J. Rogers, Ann. N.Y. Acad. Sci., 1027, 474-494 (2004).

41. A. Hanlon, D.M. Matson and R. Hyers, Philosophical Magazine Letters, 86, 165-174 (2006).

42. W. Löser, R. Hermann, T. Woodcock, J. Fransaer, M. Krivilyov, L. Gránásy, T. Pusztai, G. Toth, D. Herlach, D. Holland-Moritz, M. Kolbe and T. Volkmann, J. Jpn. Soc. Micrograv. Appl., 25, 319 324 (2008).

43. H.J. Fecht, S. Schneider, R. Wunderlich, L. Battezzati, C. Papandrea, M. Palumbo, I. Egry, K. Mills, P. Quested, R. Brooks, G. Giuranno, R. Novakovic, A. Passerone, E. Ricci, S. Seetharaman, R. Aune, B. Vinet and J. Garandet, Met. Fisica, 3, 47-51 (2005). 


\section{.}

